\title{
No Speculation under Expectations in Awareness
}

\author{
Ken Horie $^{1}$ and Takashi Matsuhisa ${ }^{2 \star}$ \\ 1 Advanced Course of Electronic and Computer Engineering, Ibaraki National \\ College of Technology \\ 2 Department of Liberal Arts and Sciences, Ibaraki National College of Technology, \\ Nakane 866, Hitachinaka-shi, Ibaraki 312-8508, Japan. \\ mathisa@ge.ibaraki-ct.ac.jp
}

\begin{abstract}
We present the extended notion of pure exchange economy under uncertainty, called an economy with awareness structure, where each trader makes decision under his/her awareness and belief and has strictly monotone preferences. We show the no speculation theorem: If the initial endowment is ex-ante Pareto optimal then there exists no other expectations equilibrium in awareness for any price.
\end{abstract}

Keywords: Pure exchange economy, Awareness, Belief, No speculation, Expectations equilibrium, Ex-ante Pareto optimum.

\section{Introduction}

This article relates economies and distributed knowledge. The purposes are the two points: First we present an extended notion of economy under uncertainty, called an economy with awareness structure, where each trader makes decision in his/her awareness and belief under incomplete information. Secondly we show the following 'no trade theorem' under generalized expectations equilibrium in the extended economy:

No speculation theorem. In a pure exchange economy under uncertainty, the traders are assumed to have an awareness structure and to have strictly monotone preferences. If the initial endowment is ex-ante Pareto optimal then there exists no other expectations equilibrium in awareness.

Recently researchers in such fields as economics, AI, and computer science have become interested in reasoning of belief and knowledge. There are pragmatic concerns about the relationship between knowledge (belief) and actions. Of most interest to us is the emphasis on situations involving the distributed knowledge (belief) of a group of agents rather than that of a single agent. At the heart of any analysis of such situations as a conversation, a bargaining session or a

\footnotetext{
* Corresponding author. Partially supported by the Grant-in-Aid for Scientific Rese$\operatorname{arch}(\mathrm{C})(2)($ No.14540145) in the Japan Society for the Promotion of Sciences. Dedicated to Professor Shoji Koizumi on the occasion of his 80th birthday
} 
protocol run by processes is the interaction between agents. A agent in a group must take into account not only events that have occurred in the world but also the knowledge of the other agents in the group.

As for as concerned in economic situations, many authors have shown that there can be no speculation in an economy under uncertainty (e.g Kreps [7; Milgrom and Stokey [12]; Morris [13] and other:11). The serious limitations of their analysis are to assume 'partition' structure as information the traders receive, and to assume common-knowledge on traders' willingness to trade. From the epistemic point of view, the partition structure represents the traders' knowledge satisfying the postulates: 'Truth' $\mathbf{T}$ (what is known is true), the 'positive introspection' $\mathbf{4}$ (that we know what we do) and the 'negative introspection' 5 (that we know what we do not know). The postulate $\mathbf{5}$ is indeed so strong that describes the hyper-rationality of traders, and thus it is particularly objectionable. Also is the common knowledge assumption because the notion of common knowledge is defined by an infinite recursion of all of individual knowledge.

This raises the question to what extent results as the no speculation theorem depend on both common knowledge and the information partition structure (or the equivalent postulates of knowledge). The answer is the results which strengthen the Milgrom and Stokey's theorem. In this article we weaken the conditions: symmetry, reflexivity, transitivity and common-knowledge. As has already been pointed out in Geanakoplos (1989), this relaxation can potentially yield important results in a world with imperfectly Bayesian agents.

This article is organized as follows: In Section 2 we present our formal model: First we recall belief and awareness together with the associated information structure. Secondly we give economies with awareness structure, and we extend the notion of rational expectations equilibrium for economies with incomplete information to the economies with awareness structure. In Section 3 we explicitly state the no speculation theorem as above and prove it. Finally we discuss related results and conclude by giving some remarks on the assumptions of our results.

\section{The Model}

Let $\Omega$ be a no-empty finite set called a state space, $T$ a set of finitely many at least two traders $\{1,2, \ldots, t, \ldots n\}(n \geq 2)$, and let $2^{\Omega}$ denote the field $2^{\Omega}$ that is the family of all subsets of $\Omega$. Each member of $2^{\Omega}$ is called an event and each element of $\Omega$ called a state.

\subsection{Awareness and Belief}

We present a model of awareness according to Matsuhisa and Usami $[112$. $A$ belief structure is a tuple $\left\langle\Omega,\left(B_{t}\right)_{t \in N}\right\rangle$ in which $B_{t}: 2^{\Omega} \rightarrow 2^{\Omega}$ is trader $t$ 's

\footnotetext{
${ }^{1}$ The references cited in Fudenberg and Tirole [5], footnote 3, p.543

${ }^{2}$ This model follows from E. Dekel, B. L. Lipman and A. Rustichini [3. A different approach of awareness models is discussed in R. Fagin, J.Y. Halpern, Y. Moses and M.Y. Vardi [4].
} 
belief operator. The interpretation of the event $B_{t} E$ is that ' $t$ believes $E$.' An awareness structure is a tuple $\left\langle\Omega,\left(A_{t}\right)_{t \in T},\left(B_{t}\right)_{t \in T}\right\rangle$ in which $\left\langle\Omega,\left(B_{t}\right)_{t \in T}\right\rangle$ is a belief structure and $A_{t}$ is $t$ 's awareness operator on $2^{\Omega}$ such that Axiom PL (axiom of plausibility) is valid:

PL $\quad B_{t} E \cup B_{t}\left(\Omega \backslash B_{t} E\right) \subseteq A_{t} E \quad$ for every $E$ in $2^{\Omega}$.

The interpretation of $A_{t} E$ is that ' $t$ is aware of $E$.' The axiom $\mathbf{P L}$ says that $t$ is aware of $E$ if he believes it or if he believes that he does not believe it. An event $E$ is said to be $t$ 's evident belief if $T \subseteq B_{t} T$. We can think of it as embodying the essence of what is involved in $t$ making his/her direct observations.

\subsection{Associated Information Structure}

M. Bacharach [1] introduces the strong epistemic model that coincides with the Kripke semantics corresponding to the modal logic S5.3 Further he defines the information partition induced from the knowledge operator of the epistemic model 4 Following his line we generalize the notion of information partition as follows.

Definition 1. The associated information structure $\left(P_{t}\right)_{t \in T}$ with an awareness structure $\left\langle\Omega,\left(A_{t}\right),\left(B_{t}\right)\right\rangle$ is the class of $t$ 's associated information function $P_{t}$ of $\Omega$ into $2^{\Omega}$ defined by $P_{t}(\omega)=\bigcap_{E \in 2^{\Omega}}\left\{E \mid \omega \in E \subseteq B_{t} E\right\}$. (If there is no event $E$ for which $\omega \in E \subseteq B_{t} E$ then we take $P_{t}(\omega)$ to be no-defined.) The domain of $P_{t}$ denoted by $\operatorname{Dom}\left(P_{t}\right)$ is the set of all the states at which $P_{t}$ is defined.

We call $P_{t}(\omega)$ t's evidence set at $\omega$, which is interpreted as the basis for all $t$ 's evident beliefs. This is because each $t$ 's evident belief $E$ is decomposed into a union of all evidence sets contained in $E$.

We note that each mapping $P_{t}$ is reflexive on its domain in the below sense: The mapping $P_{t}: \Omega \rightarrow 2^{\Omega}$ is said to be reflexive if

Ref $\omega \in P_{t}(\omega)$ for every $\omega \in \operatorname{Dom}\left(P_{t}\right)$,

and it is said to be transitive if:

Trn $\xi \in P_{t}(\omega)$ implies $P_{t}(\xi) \subseteq P_{t}(\omega)$ for any $\xi, \omega \in \operatorname{Dom}\left(P_{t}\right)$.

Furthermore $P_{t}$ is said to be symmetric if:

3 The strong epistemic model is a tuple $\left\langle\Omega,\left(K_{t}\right)_{t \in T}\right\rangle$, in which $t$ 's knowledge operator $K_{t}: 2^{\Omega} \rightarrow 2^{\Omega}$ satisfies the five postulates: For every $E, F$ of $2^{\Omega}$,
$\mathbf{N} K_{t} \Omega=\Omega$;
$\mathbf{K} \quad K_{t}(E \cap F)=K_{t} E \cap K_{t} F$
T $\quad K_{t} F \subseteq F$
$4 K_{t} F \subseteq K_{t} K_{t} F$;
$\mathbf{5} \Omega \backslash K_{t} F \subseteq K_{t}\left(\Omega \backslash K_{t} F\right)$.

4 's information partition $P_{t}$ induced from the knowledge operator $K_{t}$ is defined by $P_{t}(\omega)=\bigcap_{T \in 2^{\Omega}}\left\{T \in 2^{\Omega} \mid \omega \in K_{t} T\right\}$. 
Sym $\xi \in P_{t}(\omega)$ implies $P_{t}(\xi) \ni \omega$ for any $\xi, \omega \in \operatorname{Dom}\left(P_{t}\right)$.

Remark 1. The strong epistemic model can be interpreted as the awareness structure $\left\langle\Omega,\left(A_{t}\right),\left(B_{t}\right)\right\rangle$ such that $\left(\Omega, B_{t}\right)$ is the Kripke semantics corresponding to the logic S5. In this situation it is easily verified that $A_{t}$ must be the trivial operator; i.e. $A_{t}(F)=\Omega$ for every $F \in 2^{\Omega}$. We note that for each $t$, the associated information function $P_{t}$ is a partition of the state space $\Omega$ with $\operatorname{Dom}\left(P_{t}\right)=\Omega$ in the strong epistemic model.

\subsection{Economy with Awareness Structure}

A pure exchange economy under uncertainty is a tuple $\left\langle T, \Omega, \mathbf{e},\left(U_{t}\right)_{t \in T},\left(\pi_{t}\right)_{t \in T}\right\rangle$ consisting of the following structure and interpretations: There are $l$ commodities in each state of the state space $\Omega$, and it is assumed that $\Omega$ is finite and that the consumption set of trader $t$ is $\mathbb{R}_{+}^{l} ; \mathbf{e}(t, \cdot): T \times \Omega \rightarrow \mathbb{R}_{+}^{l}$ is $t$ 's initial endowment; $U_{t}: \mathbb{R}_{+}^{l} \times \Omega \rightarrow \mathbb{R}$ is $t$ 's von-Neumann and Morgenstern utility function; $\pi_{t}$ is a subjective prior on $\Omega$ for a trader $t \in T$. For simplicity it is assumed that $\left(\Omega, \pi_{t}\right)$ is a finite probability space with $\pi_{t}$ full support5for all $t \in T$.

Definition 2. A pure exchange economy with awareness structure is a structure $\mathcal{E}^{A}=\left\langle\mathcal{E},\left(A_{t}\right)_{t \in T},\left(B_{t}\right)_{t \in T},\left(P_{t}\right)_{t \in T}\right\rangle$, in which $\mathcal{E}$ is a pure exchange economy such that $\left\langle\Omega,\left(A_{t}\right)_{t \in T},\left(B_{t}\right)_{t \in T},\left(P_{t}\right)_{t \in T}\right\rangle$ is an awareness structure with $\left(P_{t}\right)_{t \in T}$ the associated information structure. By the domain of the economy $\mathcal{E}^{A}$ we mean $\operatorname{Dom}\left(\mathcal{E}^{A}\right)=\cap_{t \in T} \operatorname{Dom}\left(P_{t}\right)$. We always assume the below condition:

A-0 $\operatorname{Dom}\left(\mathcal{E}^{A}\right) \neq \emptyset$.

Remark 2. An economy under asymmetric information is an economy $\mathcal{E}^{A}$ in which $\left\langle\Omega,\left(A_{t}\right)_{t \in T},\left(B_{t}\right)_{t \in T}\right\rangle$ is given by the strong epistemic model by Bacha$\operatorname{rach}[1]$.

We denote by $\mathcal{F}_{t}$ the field generated by $\left\{P_{t}(\omega) \mid \omega \in \Omega\right\}$ and denote by $\Pi_{t}(\omega)$ the atom containing $\omega \in \Omega$. We denote by $\mathcal{F}$ the join of all $\mathcal{F}_{t}(t \in T)$; i.e. $\mathcal{F}=\vee_{t \in T} \mathcal{F}_{t}$, and denote by $\{\Pi(\omega) \mid \omega \in \Omega\}$ the set of all atoms $\Pi(\omega)$ containing $\omega$ of the field $\mathcal{F}=\vee_{t \in T} \mathcal{F}_{t}$.

We shall often refer to the following conditions: For every $t \in T$,

A-1 $t$ 's endowment $\mathbf{e}(t, \cdot)$ is $\mathcal{F}_{t}$-measurable with $\sum_{t \in T} \mathbf{e}(t, \omega)>0$ for all $\omega \in \Omega$.

A-2 For each $x \in \mathbb{R}_{+}^{l}$, the function $U_{t}(x, \cdot)$ is $\mathcal{F}_{t}$-measurable.

A-3 For each $\omega \in \Omega$, the function $U_{t}(\cdot, \omega)$ is continuous, increasing, strictly quasi-concave and non-saturated 6 on $\mathbb{R}_{+}^{l}$.

A-4 For all $t \in T, \operatorname{Dom}\left(\mathcal{E}^{A}\right)=\operatorname{Dom}\left(P_{t}\right)$

${ }^{5}$ That is, $\pi_{t}(\omega) \neq 0$ for every $\omega \in \Omega$.

${ }^{6}$ That is, for any $x \in \mathbb{R}_{+}^{l}$ there exists an $x^{\prime} \in \mathbb{R}_{+}^{l}$ such that $U_{t}\left(x^{\prime}, \omega\right)>U_{t}(x, \omega)$. 
An assignment $\mathbf{x}$ is a mapping from $T \times \Omega$ into $\mathbb{R}_{+}^{l}$ such that for every $\omega \in \Omega$ and for each $t \in T$, the function $\mathbf{x}(t, \cdot)$ is at most $\mathcal{F}$-measurable. We denote by $\mathcal{A s s}\left(\mathcal{E}^{A}\right)$ the set of all assignments for the economy $\mathcal{E}^{A}$. By an allocation we mean an assignment a such that for every $\omega \in \Omega, \sum_{t \in T} \mathbf{a}(t, \omega) \leq \sum_{t \in T} \mathbf{e}(t, \omega)$. We denote by $\mathcal{A} l c\left(\mathcal{E}^{A}\right)$ the set of all allocations.

We introduce the revised notion of trader's expectation of utility in $\mathcal{E}^{A}$. By $t$ 's ex-ante expectation we mean $\mathbf{E}_{t}\left[U_{t}(\mathbf{x}(t, \cdot)]:=\sum_{\omega \in \operatorname{Dom}\left(P_{t}\right)} U_{t}(\mathbf{x}(t, \omega), \omega) \pi_{t}(\omega)\right.$ for each $\mathbf{x} \in \mathcal{A} s s\left(\mathcal{E}^{A}\right) 7$ The interim expectation $\mathbf{E}_{t}\left[U_{t}\left(\mathbf{x}(t, \cdot) \mid P_{t}\right]\right.$ is defined by

$$
\mathbf{E}_{t}\left[U_{t}\left(\mathbf{x}(t, \cdot) \mid P_{t}\right](\omega):=\sum_{\xi \in \operatorname{Dom}\left(P_{t}\right)} U_{t}(\mathbf{x}(t, \xi), \xi) \pi_{t}\left(\{\xi\} \cap A_{t}(\{\xi\}) \mid P_{t}(\omega)\right) 8\right.
$$

An allocation $\mathbf{x}$ in an economy $\mathcal{E}^{A}$ is said to be ex-ante Pareto-optimal if there is no allocation a such that $\mathbf{E}_{t}\left[U_{t}(\mathbf{a}(t, \cdot)] \geq \mathbf{E}_{t}\left[U_{t}(\mathbf{x}(t, \cdot)]\right.\right.$ for every $t \in T$, with at least one inequality strict.

\subsection{Expectations Equilibrium in Awareness}

A price system is a non-zero $\mathcal{F}$-measurable function $p: \Omega \rightarrow \mathbb{R}_{+}^{l}$. We denote $\Delta(p)$ the partition induced by $p$, and denote by $\sigma(p)$ the field generated by $\Delta(p)$. The budget set of a trader $t$ at a state $\omega$ for a price system $p$ is defined by $B_{t}(\omega, p):=\left\{x \in \mathbb{R}_{+}^{l} \mid p(\omega) \cdot x \leq p(\omega) \cdot \mathbf{e}(t, \omega)\right\}$.

Let $\Delta(p) \cap P_{t}: \operatorname{Dom}\left(P_{t}\right) \rightarrow 2^{\Omega}$ be defined by $\left(\Delta(p) \cap P_{t}\right)(\omega):=\Delta(p)(\omega) \cap$ $P_{t}(\omega)$; it is plainly observed that the mapping $\Delta(p) \cap P_{t}$ satisfies Ref. We denote by $\sigma(p) \vee \mathcal{F}_{t}$ the smallest $\sigma$-field containing both the fields $\sigma(p)$ and $\mathcal{F}_{t}$, and denote by $\Pi_{t}(p)(\omega)$ the atom containing $\omega$. We note that $\Pi_{t}(p)(\omega)=(\Delta(p) \cap$ $\left.\Pi_{t}\right)(\omega)$. We shall give the extended notion of rational expectations equilibrium.

Definition 3. An expectations equilibrium for an economy $\mathcal{E}^{A}$ under reflexive information structure is a pair $(p, \mathbf{x})$, in which $p$ is a price system and $\mathbf{x}$ is an allocation satisfying the following conditions:

RE 1 For all $t \in T, \mathbf{x}(t, \cdot)$ is $\sigma(p) \vee \mathcal{F}_{t}$-measurable.

RE 2 For all $t \in T$ and for every $\omega \in \Omega, \mathbf{x}(t, \omega) \in B_{t}(\omega, p)$.

RE 3 For all $t \in T$, if $\mathbf{y}(t, \cdot): \Omega \rightarrow \mathbb{R}_{+}^{l}$ is $\sigma(p) \vee \mathcal{F}_{t}$-measurable with $\mathbf{y}(t, \omega) \in B_{t}(\omega, p)$ for all $\omega \in \Omega$, then $\mathbf{E}_{t}\left[U_{t}(\mathbf{x}(t, \cdot)) \mid \Delta(p) \cap P_{t}\right](\omega) \geq$ $\mathbf{E}_{t}\left[U_{t}(\mathbf{y}(t, \cdot)) \mid \Delta(p) \cap P_{t}\right](\omega)$ pointwise on $\operatorname{Dom}\left(P_{t}\right)$.

RE 4 For every $\omega \in \Omega, \sum_{t \in T} \mathbf{x}(t, \omega)=\sum_{t \in T} \mathbf{e}(t, \omega)$.

The allocation $\mathbf{x}$ in $\mathcal{E}^{A}$ is called an expectations equilibrium allocation for $\mathcal{E}^{A}$.

\footnotetext{
${ }^{7}$ Where it is noted that $\xi \in \Omega$ in the summation runs over the domain of $P_{t}$.

${ }^{8}$ It should be noted that we use not the usual notion of posterior $\pi_{t}\left(\{\xi\} \mid P_{t}(\omega)\right)$ but the revised one $\pi_{t}\left(\{\xi\} \cap A_{t}(\{\xi\}) \mid P_{t}(\omega)\right)$. For the discussion why this improvement of the notion of posterior is needed, see Matsuhisa and Usami [1] (Section 4).
} 
We denote by $R E\left(\mathcal{E}^{A}\right)$ the set of all the rational expectations equilibria of a pure exchange economy $\mathcal{E}^{A}$ with awareness structure, and denote by $\mathcal{R}\left(\mathcal{E}^{A}\right)$ the set of all the expectations equilibrium allocations for the economy. We can establish the existence theorem of the expectations equilibrium for a pure exchange economy $\mathcal{E}^{A}$ with awareness structure.

Theorem 1. Let $\mathcal{E}^{A}$ be a pure exchange economy with awareness structure satisfying the conditions $\mathbf{A - 1}, \mathbf{A - 2}, \mathbf{A - 3}$ and $\mathbf{A - 4}$. Then there exists an expectations equilibrium for the economy; i.e., $R E\left(\mathcal{E}^{A}\right) \neq \emptyset$.

Proof. Can be given in the same line of Matsuhisa [8].

\section{No Speculation Theorem}

We can now state the main theorem explicitly and prove it.

Theorem 2. Let $\mathcal{E}^{A}$ be a pure exchange economy with awareness structure satisfying the conditions A-1, A-2, A-3 and A-4. Suppose that the initial endowment $\mathbf{e}$ is ex-ante Pareto optimal in $\mathcal{E}^{A}$. If $(p, \mathbf{x})$ is an expectations equilibrium for $\mathcal{E}^{A}$ for some price system $p$ then $\mathbf{x}=\mathbf{e}$.

Before proceeding with the proof we shall show the below propositions: Let $\mathcal{E}^{A}(\omega)$ denote the economy with complete information $\left\langle T,(\mathbf{e}(t, \omega))_{t \in T},\left(U_{t}(*, \omega)\right)_{t \in T}\right\rangle$ for each $\omega \in \Omega$. We set by $W\left(\mathcal{E}^{A}(\omega)\right)$ the set of all the competitive equilibria for $\mathcal{E}^{A}(\omega)$, and we denote by $\mathcal{W}\left(\mathcal{E}^{A}(\omega)\right)$ the set of all the competitive equilibrium allocations for $\mathcal{E}^{A}(\omega)$.

Proposition 1. Let $\mathcal{E}^{A}$ be a pure exchange economy with awareness structure satisfying the conditions $\mathbf{A - 1}, \mathbf{A - 2}, \mathbf{A - 3}$ and $\mathbf{A - 4}$. The set of all expectations equilibrium allocations for $\mathcal{R}\left(\mathcal{E}^{A}\right)$ coincides with the set of all the assignments $\mathbf{x}$ such that $\mathbf{x}(\cdot, \omega)$ is a competitive equilibrium allocation for the economy with complete information $\mathcal{E}^{A}(\omega)$ for all $\omega \in \operatorname{Dom}\left(\mathcal{E}^{A}\right)$; i.e.,

$$
\begin{aligned}
\mathcal{R}\left(\mathcal{E}^{A}\right)=\left\{\mathbf{x} \in \mathcal{A l c}\left(\mathcal{E}^{A}\right) \mid\right. & \text { There is a price system } p \text { such that } \\
& \left.(p(\omega), \mathbf{x}(\cdot, \omega)) \in W\left(\mathcal{E}^{A}(\omega)\right) \text { for all } \omega \in \operatorname{Dom}\left(\mathcal{E}^{A}\right)\right\} .
\end{aligned}
$$

Proof. 9 In view of the conditions A-1, A-2 and A-3, it follows from the existence theorem of a competitive equilibrium that for each $\omega \in \Omega$, there exists a competitive equilibrium $\left(p^{*}(\omega), \mathbf{x}^{*}(\cdot, \omega)\right) \in W\left(\mathcal{E}^{A}(\omega)\right)$ (C.f.; Debreu [2]). We take a set of strictly positive numbers $\left\{k_{\omega}\right\}_{\omega \in \Omega}$ such that $k_{\omega} p^{*}(\omega) \neq k_{\xi} p^{*}(\xi)$ for any $\omega \neq \xi$. We define the pair $(p, \mathbf{x})$ as follows: For each $\omega \in \Omega$ and for all $\xi \in \Pi(\omega)$, $p(\xi):=k_{\omega} p^{*}(\omega)$ and $\mathbf{x}(t, \xi):=\mathbf{x}^{*}(t, \omega)$. It is noted that $\mathbf{x}(\cdot, \xi) \in W\left(\mathcal{E}^{A}(\omega)\right)$ because $\mathcal{E}^{A}(\xi)=\mathcal{E}^{A}(\omega)$, and we note that $\Delta(p)(\omega)=\Pi(\omega)$.

We shall verify that $(p, \mathbf{x})$ is an expectations equilibrium for $\mathcal{E}^{A}$ : In fact, it is easily seen that $p$ is $\mathcal{F}$-measurable with $\Delta(p)(\omega)=\Pi(\omega)$ and that $\mathbf{x}(t, \cdot)$ is

\footnotetext{
${ }^{9}$ The proof is given in the similar line of Matsuhisa, Ishikawa and Hoshino [10] (Proposition 2).
} 
$\sigma(p) \vee \mathcal{F}_{t}$-measurable, so $\mathbf{R E} \mathbf{1}$ is valid. Because $\left(\Delta(p) \cap P_{t}\right)(\omega)=\Pi(\omega)$ for every $\omega \in \operatorname{Dom}\left(\mathcal{E}^{A}\right)$, it can be plainly observed that $\mathbf{x}(t, \cdot)$ satisfies $\mathbf{R E} \mathbf{2}$, and it follows from A-2 that for all $t \in T, \mathbf{E}_{t}\left[U_{t}(\mathbf{x}(t, \cdot)) \mid \Delta(p) \cap P_{t}\right](\omega)=U_{t}(\mathbf{x}(t, \omega), \omega)$

On noting that $\mathcal{E}^{A}(\xi)=\mathcal{E}^{A}(\omega)$ for any $\xi \in \Pi(\omega)$, it is plainly observed that $(p(\omega), \mathbf{x}(t, \omega))=\left(k_{\omega} p^{*}(\omega), \mathbf{x}^{*}(t, \omega)\right)$ is also a competitive equilibrium for $\mathcal{E}^{A}(\omega)$ for every $\omega \in \operatorname{Dom}\left(\mathcal{E}^{A}\right)$, and it can be observed by the above equation that $\mathbf{R E}$ $\mathbf{3}$ is valid for $(p, \mathbf{x})$, in completing the proof.

Proposition 2. Let $\mathcal{E}^{A}$ be a pure exchange economy with awareness structure satisfying the conditions $\mathbf{A - 1}, \mathbf{A - 2}, \mathbf{A - 3}$ and $\mathbf{A}-\mathbf{4}$. Then an allocation $\mathbf{x}$ is exante Pareto optimal if it is an expectations equilibrium allocation for $\mathcal{E}^{A}$ relative to a price system.

Proof. Let $(p, \mathbf{x})$ be an expectations equilibrium for $\mathcal{E}^{A}$. It follows from Proposition 1 1 that $(p(\omega), \mathbf{x}(\cdot, \omega))$ is a competitive equilibrium for the economy $\mathcal{E}^{A}(\omega)$ at each $\omega \in \operatorname{Dom}\left(\mathcal{E}^{A}\right)$. Therefore in viewing the well known fundamental theorem of welfare in the economy with complete information $\mathcal{E}^{A}(\omega)$, we can plainly observe that for each $t$ and for all $\omega \in \operatorname{Dom}\left(\mathcal{E}^{A}\right)=\operatorname{Dom}\left(P_{t}\right), \mathbf{x}(\cdot, \omega)$ is Pareto optimal in $\mathcal{E}^{A}(\omega)$, and we conclude that $\mathbf{x}$ is ex-ante Pareto optimal.

Proof of Theorem 2. Let $(p, \mathbf{x}) \in R E\left(\mathcal{E}^{A}\right)$. It follows from Proposition 2 that $\mathbf{x}$ is ex-ante Pareto optimal in $\mathcal{E}^{A}$. Suppose to the contrary that $\mathbf{x} \neq \mathbf{e}$. Since e is ex-ante Pareto optimal in $\mathcal{E}^{A}$ it can be observed that there exist an $s \in S$ such that $\mathbf{E}_{s}\left[U_{s}(\mathbf{e}(s, \cdot))\right]>\mathbf{E}_{s}\left[U_{s}(\mathbf{x}(s, \cdot))\right]$. Therefore, it can be plainly verified that for some $\omega_{0} \in \operatorname{Dom}\left(P_{s}\right), U_{s}\left(\mathbf{e}\left(s, \omega_{0}\right), \omega_{0}\right)>U_{s}\left(\mathbf{x}\left(s, \omega_{0}\right), \omega_{0}\right)$. On the other hand, it follows from Proposition 1 that $\left(p\left(\omega_{0}\right), \mathbf{x}\left(\cdot, \omega_{0}\right)\right) \in W\left(\mathcal{E}^{A}\left(\omega_{0}\right)\right)$, thus $U_{s}\left(\mathbf{x}\left(s, \omega_{0}\right), \omega_{0}\right)>U_{s}\left(\mathbf{e}\left(s, \omega_{0}\right), \omega_{0}\right)$ in contradiction. This completes the proof.

\section{Related Results and Remarks}

We shall discuss related results: Welfare in economics and Core equivalence. Finally we give some remarks about the conditions A-1 to A-4.

The converse of Propositions 2 can be proved by the similar way in Matsuhisa and Ishikawa 9] (Proposition 4). Therefore we can characterize welfare under the expectations equilibrium for the economy $\mathcal{E}^{A}$ : Let $\mathcal{E}^{A}$ be a pure exchange economy with awareness structure satisfying the conditions $\mathbf{A - 1}, \mathbf{A - 2}, \mathbf{A}-\mathbf{3}$ and $\mathbf{A - 4}$. An allocation is ex-ante Pareto optimal if and only if it is an expectations equilibrium allocation for $\mathcal{E}^{A}$ relative to some price system.

Matsuhisa [8] introduces the notion of ex-post core in the economy for modal logic KT. Based on the article of Matsuhisa, Ishikawa and Hoshino [10 he establishes the core equivalence theorem in the economy equipped with nonatomic measure on the traders space: The ex-post core in the economy for $\mathbf{K T}$ coincides with the set of all its rational expectations equilibria. We can extend 
the core equivalence theorem into the economy with awareness structure, and we shall report it in near future.

It well ends some remarks about the auxiliary assumptions $\mathbf{A - 1}$ to $\mathbf{A - 4}$ in results in this article. Could we prove the theorems without four conditions $\mathbf{A - 1}$, A-2 and A-3. The answer is no vein. The suppression of any of these assumptions renders the existence theorem of expectations equilibrium for $\mathcal{E}^{A}$ (Theorem 1 ) vulnerable to the discussion and the example proposed in Remarks 4.6 of Matsuhisa and Ishikawa [9]. Could we prove the theorems removing out A-4? The answer is no again. If $t$ 's associated information function $P_{t}$ does not satisfy Ref then his/her expectation with respect to a price cannot be defined at a state because it is possible that $\Delta(p)(\omega) \cap P_{t}(\omega)=\emptyset$ for some $\omega \in \Omega$.

\section{References}

1. Bacharach,M.: Some extensions of a claim of Aumann in an axiomatic model of knowledge. Journal of Economic Theory 37 (1985) 167-190.

2. Debreu, G.: Existence of competitive equilibrium. In Arrow, K.J. and Intriligator, M.D., (eds): Handbook of Mathematical Economics, Volume 2. North-Holland Publishing Company, Amsterdam (1982) 697-744.

3. Dekel,E., Lipman,B.L. and Rustichini,A.: Standard state-space models preclude unawareness. Econometrica 66 (1998) 159-173.

4. Fagin,R., Halpern,J.Y., Moses,Y. and Vardi, M.Y.: Reasoning about Knowledge. The MIT Press, Cambridge, Massachusetts, London, England, 1995.

5. Fudenberg, D. and Tirole, J., Game Theory. MIT Press, Cambridge USA, xxii+579, 1991

6. Geanakoplos, J.: Game theory without partitions, and applications to speculation and consensus, Cowles Foundation Discussion Paper No.914 (1989) (Available in http://cowles.econ.yale.edu)

7. Kreps, D.: A note on fulfilled expectations equilibrium, Journal of Economic Theory 14 (1977) 32-44.

8. Matsuhisa, T.: Core equivalence in economy for modal logic, in P.M.A. Sloot, D. Abramson et al. (eds): Computational Science-ICCS 2003, Proceedings, Springer Lecture Notes of Computer Science, Vol. 2658 (2003) 74-83.

9. Matsuhisa, T. and Ishikawa, R.: Rational expectations can preclude trades. Preprint, Hitotsubashi Discussion Paper Series 2002-1 (2002) (Available in http://wakame.econ.hit-u.ac.jp/).

10. Matsuhisa, T., Ishikawa, R. and Hoshino, Y., 2002. Core equivalence in economy under generalized information. Working paper. Hitotsubashi Discussion Paper Series No.2002-12 (2002) (Available in http://wakame.econ.hit-u.ac.jp/)

11. Matsuhisa,T. and Usami,S.-S.: Awareness, belief and agreeing to disagree. Far East Journal of Mathematical Sciences 2 (2000) 833-844.

12. Milgrom, P. and Stokey, N.: Information, trade and common knowledge, Journal of Economic Theory 26 (1982) 17-27.

13. Morris, S.: Trade with heterogeneous prior beliefs and asymmetric information, Econometrica 62 (1994) 1327-1347. 